\title{
THE COMPUTATION OF THE INDEX OF A MORSE FUNCTION AT A CRITICAL POINT
}

TAKIS SAKKALIS

\author{
Department of Mathematical Sciences \\ New Mexico State University \\ Las Cruces, NM 88003 \\ and
}

IBM T.J. Watson Research Center

P.O. Box 218

Yorktown Heights, NY 10598

(Received April 24, 1987 and in revised form February 12, 1988)

ABSTRACT. A theoretical approach in computing the index of a Morse function at a critical point on a real non-singular hypersurface $V$ is given. As a consequence the Euler characteristic of $V$ is computed. In the case where the hypersurface is polynomial and compact, a procedure is given that finds a linear function $\ell$, whose restriction $\left.\ell\right|_{V}$, is a Morse function on $V$.

Key Words and Phrases. Morse function, critical point, index. 1980 Ams Subject Classification Codes. Primary 58, Secondary 55.

1. INTRODUCTION.

Let $f\left(x_{1}, \ldots, x_{n}\right)$ be a real $C^{\infty}$ function, and set

$$
V=\left\{\left(x_{1}, \ldots, x_{n}\right) \in \mathbf{R}^{n} \mid f\left(x_{1}, \ldots, x_{n}\right)=0\right\} .
$$

Suppose $V$ is non-singular in $\mathbf{R}^{n}$. Furthermore, let $g\left(x_{1}, \ldots, x_{n}\right)$ be a real function whose restriction $\left.g\right|_{V}$, on $V$, is a Morse function. Then we will first give a theoretical approach of how the Morse index of $\left.g\right|_{V}$ at a critical point $a$ can be computed. Using the above data, we can also compute the Euler characteristic, $\chi(V)$, of $V$.

Finally, in the case where $f$ is a polynomial, we will say how we can obtain a polynomial function $g$, whose restriction $\left.g\right|_{V}$, has no degenerate critical points on $V$. 


\section{Tile Basic Result.}

We first recall some well known results from Morse Theory [3]. For $A$ a $k \times k$ real non-singular symmetric matrix, we denote by the index of $A, \imath(A)$, the number of negative eigenvalues of $A$. Using the above definition, we may define the index of a Morse function at a critical point. Let $\mu: I V \rightarrow \mathbf{R}$ be a real Morse function on a $r$-manifold $W$, and also let $w \in W$ be a critical point of $\mu$. For $u_{1}, \ldots, u_{r}$ local coordinates on $W$ around $w$ we can form the Hessian matrix of $\mu$ with respect to $u_{1}, \ldots, u_{r}, H \mu(u), H \mu(u)=\left(\frac{\partial^{2} \mu}{\partial u_{,} \partial u_{\jmath}}\right), 1 \leq \imath, \jmath \leq r$. Although the Hessian matrix $H \mu(u)$ depends on the particular coordinates $u$, its index does not. We then define:

Definition 1. The index of $\mu$ at the critical point $w, i(w)=i(H \mu(u))$ for some coordinates $u$ around $w$.

Let us now fix some notation. For $R\left(x_{1}, \ldots, x_{n}\right)$ a real $C^{\infty}$ function, we denote by $R_{\imath}=\frac{\partial R}{\partial x_{1}}, i=1, \ldots, n, R_{\imath \jmath}=\frac{\partial^{2} R}{\partial x_{1} \partial x_{j}}, i, j=1, \ldots, n$.

Let $a$ be a critical point of $\left.g\right|_{V}$. Without loss of generality, we may assume that $f_{n}(a) \neq 0$. Then using the Implicit Function Theorem we may "solve" the equation $f\left(x_{1}, \ldots, x_{n}\right)=0$ for $x_{n}$, i.e. near $a, V$ can be thought as the graph of $x_{n}=x_{n}\left(x_{1}, \ldots, x_{n-1}\right)$, and, therefore, $x_{1}, \ldots, x_{n-1}$ are local coordinates for $V$ near $a$. If we differentiate the equation $f\left(x_{1}, \ldots, x_{n}\right)=0$ twice, and evaluate at $a$, we get:

(I) $0=f_{\imath \jmath}+f_{n_{\imath}} \cdot x_{n,}+f_{n \jmath} \cdot x_{n_{\mathrm{\imath}}}+f_{n n} \cdot x_{n_{\jmath}} \cdot x_{n_{\mathrm{\imath}}}+f_{n} \cdot x_{n_{\imath},}, i, j=1, \ldots, n-1$.

At $a$ again we have,

$$
g_{\mathfrak{\imath}}=\lambda f_{\mathfrak{\imath}}, i=1, \ldots, n, \lambda \in \mathbf{R} .
$$

Now $\left.g\right|_{V}$ with respect to the coordinates $x_{1}, \ldots, x_{n-1}$ becomes $Q\left(x_{1}, \ldots, x_{n-1}\right)=$ $g\left(x_{1}, \ldots, x_{n-1}, x_{n}\left(x_{1}, \ldots, x_{n-1}\right)\right)$. To compute therefore $i(a)$, it is enough to calculate the Hessian matrix $H Q\left(x_{1}, \ldots, x_{n-1}\right)$, at $a$. We have

$$
\begin{aligned}
Q_{\imath} & =g_{\imath}+g_{n} x_{n_{\imath}}, \text { and } \\
Q_{i \jmath} & =g_{\imath j}+g_{n i} \cdot x_{n \jmath}+g_{n \jmath} \cdot x_{n_{\imath}}+g_{n n} \cdot x_{n_{i}} \cdot x_{n \jmath}+g_{n} \cdot x_{n_{\imath},} .
\end{aligned}
$$

Substituting in III what $x_{n_{1}}$, is in I and taking II into account, we get

$$
Q_{\imath \jmath}=\frac{1}{f_{n}^{2}}\left(h_{i j} \cdot f_{n}^{2}-h_{n \imath} f_{\jmath} f_{n}-h_{n} f_{i} f_{n}+h_{n n} f_{\imath} f_{\jmath}\right),
$$

where $h=g-\lambda f, \lambda$ is the constant in II, and $1 \leq i, j \leq n-1$.

We computed the Hessian matrix $H Q\left(x_{1}, \ldots, x_{n-1}\right)=\left(Q_{2 \jmath}\right)$ at $a$. But, unfortunately, this matrix depends on the particular coordinates used at the point $a$. Let us now give a coordinate free matrix whose index is related to $i(a)$ in a linear manner.

Let $a, h, f$ be as before. Consider the following real $(n+1) \times(n+1)$ symmetric matrix $N$, evaluated at $a$. 


$$
N=\left(\begin{array}{cc}
0 & \nabla f \\
\nabla^{t} f & H(h)
\end{array}\right), \text { where } H(h)=h_{\imath \jmath}, i, j=1, \ldots, n
$$

The following proposition is the main result in this paper.

Proposition 1. For $a, h, f, N$ as above, $N$ is a non-singular matrix. Furthermore, $i(a)=i(N)-1$.

The proof of Proposition 1 will be in stages. First we will state some generalities and then come back to the proof.

For $A$ a $n \times n$ real symmetric matrix we associate the real bilinear form $q(x, y)=x^{t} A y$. We say that $q$ is non-degenerate if $(q(x, y)=0 \forall y) \Rightarrow x=0$. This is equivalent in saying that $A$ is an invertible matrix. Since $A$ is symmetric there exists an invertible matrix $P$ such that $P^{t} A P$ is diagonal. Furthermore, $i(A)=i\left(P^{t} A P\right)[2]$.

Suppose $A=\left(a_{i j}\right), i, j=1, \ldots, n$ is a real symmetric matrix. Let $v=\left(v_{1}, \ldots, v_{n}\right) \in \mathbf{R}^{n}$ so that $v_{n} \neq 0$. Consider the following real symmetric matrix $B$,

$$
B=\left(\begin{array}{cc}
0 & v \\
v^{t} & A
\end{array}\right) .
$$

For $e_{0}, e_{1}, \ldots, e_{n}$ the usual basis of $\mathbf{R}^{n+1}$, we have

$$
\begin{aligned}
& <B e_{0}, e_{0}>=0 \\
& <B e_{0}, \epsilon_{\imath}>=v_{\imath}, i=1, \ldots, n \\
& <B e_{i}, e_{j}>=a_{i j}, i, j=1, \ldots, n,
\end{aligned}
$$

where $<>$ denotes dot product.

Now introduce a new basis $e_{0}, \phi_{1}, \ldots, \phi_{n-1}, e_{n}$ on $\mathbf{R}^{n+1}$ so that $\phi_{i}=$ $v_{n} e_{2}-v_{2} e_{n}, i=1, \ldots, n-1$. With respect to those coordinates, the bilinear form $r(x, y)=x^{t} B y$ gets transformed to one whose matrix is $P^{t} B P$, where $P$ is the following matrix

$$
\begin{aligned}
P & =\left(\begin{array}{cccc}
1 & 0 & , \ldots, & 0 \\
0 & v_{n} & , \ldots, & 0 \\
0 & \vdots & \ddots & 0 \\
& & v_{n} & 0 \\
0 & -v_{1}, \ldots, & -v_{n-1} & 1
\end{array}\right), \text { and } P^{t} B P \text { becomes } \\
P^{t} B P & =\left(\begin{array}{ccc}
0, & 0, \ldots, 0, & v_{n} \\
0 & & \\
\vdots & \Gamma & \\
0 &
\end{array}\right), \text { where } \Gamma=\left(\gamma_{i},\right) i, j=1, \ldots, n
\end{aligned}
$$

and $\gamma_{i j}=v_{n}^{2} a_{\imath \jmath}-v_{n} v, a_{i n}-v_{\imath} v_{n} a_{n},+v_{\imath} v, a_{n n}, i, j=1, \ldots, n-1$. We then have:

Lemma 1. Suppose $\Gamma^{\prime}=\left(\gamma_{i j}\right), i, j=1, \ldots, n-1, \gamma_{i j}$ as above, is nonsingular. Then $B$ is also non-singular and furthermore

$$
i(B)=i\left(\Gamma^{\prime}\right)+1 .
$$


Proof. We observe that $\operatorname{det}\left(P^{t} B P\right) \neq 0$, since $v_{n} \neq 0$, and therefore $B$ is non-singular. Let $R$ be a real non-singular $(n-1) \times(n-1)$ matrix, so that $R^{t} \Gamma^{\prime} R$ is diagonal. Since $\Gamma^{\prime}$ is non-singular, all of the diagonal elements of $R^{t} \Gamma^{\prime} R$ are non-zero. Let $R^{\prime}$ be the following non-singular matrix

$$
R^{\prime}=\left(\begin{array}{cccc}
1, & 0, & \ldots, & 0 \\
0 & R & & \\
& & & 0 \\
0, & 0, & \ldots, & 1
\end{array}\right) .
$$

Now consider $S=R^{\prime t}\left(P^{t} B P\right) R^{\prime}, S$ has the form

$$
S=\left(\begin{array}{cccc}
0, & 0, & \ldots, & v_{n} \\
\vdots & \gamma_{1} & & b_{1} \\
& \ddots & & \vdots \\
& & \gamma_{n-1} & \\
v_{n} & b_{1}, & \ldots, & b_{n}
\end{array}\right) .
$$

Let $E_{\imath}$ be the following $(n+1) \times(n+1)$ elementary matrix.

$$
E_{\imath}=\left(\begin{array}{ccc}
I & 0, & 0 \\
0, & \frac{-b_{i}}{\gamma_{1}}, & 1
\end{array}\right)
$$

where $-\frac{b_{1}}{\gamma_{1}}$ appears in the $(i+1)^{t h}$ column for $i=1, \ldots, n-1$. Observe that each $E_{i}$ is invertible. Furthermore, a computation shows that $\prod_{\imath=1}^{n-1} E_{n-\imath}^{t} \cdot S$. $\prod_{\imath=1}^{n-1} E_{\imath}=S^{\prime}$ has the following form

$$
S^{\prime}=\left(\begin{array}{ccccc}
0, & 0, & \ldots, & 0, & v_{n} \\
0 & \gamma_{1} & & & 0 \\
\vdots & & \ddots & & \\
0 & & & \gamma_{n-1} & 0 \\
v_{n} & 0 & \ldots & 0 & b
\end{array}\right), \text { where } b=b_{n}-\sum_{\imath=1}^{n-1} \frac{b_{l}^{2}}{\gamma_{\imath}}
$$

On the other hand, $i\left(S^{\prime}\right)=i(S)$. To complete the proof of the lemma it is enough to show that

$$
i\left(S^{\prime}\right)=\#\left\{i^{\prime} s \mid \gamma_{\imath}<0\right\}+1
$$

To achieve that we look at the $\operatorname{det}\left(S^{\prime}-\lambda I\right)=(-\lambda)(b-\lambda) \prod_{i=1}^{n-1}\left(\gamma_{2}-\lambda\right)-$ $v_{n}^{2} \prod_{\imath=1}^{n-1}\left(\gamma_{i}-\lambda\right)=\prod_{z=1}^{n-1}\left(\gamma_{i}-\lambda\right) \cdot\left(\lambda^{2}-b \lambda-v_{n}^{2}\right)$. But the real roots of $\lambda^{2}-b \lambda-v_{n}^{2}$ are exactly two, one positive and one negative.

Proof of Proposition 1. With the same notation and the same change of coordinates, we take $B$ to be $N$, then $\Gamma^{\prime}$ becomes $A$.

And now Lemma 1 says

$$
i(N)=i(Q)+1=i(a)+1
$$

To compute the index, $i(N)$, of $N$ we first look at the negative zeros of $D(x)=\operatorname{det}(N-x I)$. To determine the number of negative zeros of $D(x)$ we can use the following argument: Let $d_{0}=\operatorname{g.c.d}\left(D, D^{\prime}\right), d_{1}=$ g.c.d $\left(d_{0}, d_{0}^{\prime}\right), \ldots$, and $d_{i}=\operatorname{g.c.d}\left(d_{\imath-1}, d_{\imath-1}^{\prime}\right), i=1, \ldots, k$, with $d_{k}$ constant where $D^{\prime}=\frac{d D}{d x}$, and 
$d_{i+1}^{\prime}=\frac{d d i}{d x}, i=0, \ldots, k-1$. Then we observe that

$\frac{1}{d_{k}} \cdot D=\frac{D}{d_{0}} \cdot \frac{d_{0}}{d_{1}} \cdot \frac{d_{1}}{d_{2}} \cdots \frac{d_{k-1}}{d_{k}}=\delta_{0} \cdot \delta_{1} \cdots \delta_{k}, \delta_{\jmath}=\frac{d_{\jmath-1}}{d_{\jmath}}, j=0, \ldots, k, d_{-1}=D$.

Furthermore, we note that each $\delta$, has simple roots and \# (negative roots of $D)=\sum_{j=0}^{k} \#$ (negative roots of $\delta_{\jmath}$ ). Finally we can use Sturm's Theorem to decide the number of negative zeros of each $\delta_{j}[1]$.

If $N$ happens to be nice, in the sense that no more than two consecutive principal minors of $N$ are singular, then $i(N)=$ variation of sign of the determinants of its principal minors [1].

The computation of the Euler characteristic, $\chi(V)$, of $V$ does not require the computation of the index of $N$, but rather the sign of its determinant. We have

$$
\chi(V)=\sum_{\substack{p \text { is a critical } \\ \text { point of } g|v|}}(-1)^{i(p)}
$$

But $(-1)^{i(p)}=\operatorname{sign} \operatorname{det}(M)(p)=-\operatorname{sign} \operatorname{det}(N)(p)$.

Therefore, $\chi(V)=-\sum_{p} \operatorname{sign} \operatorname{det}(N)(p)$.

\section{A Theoretical Procedure.}

From now on suppose that $f$ is a polynomial of even degree and $V=\{f=0\}$ is compact and non-singular in $\mathbf{R}^{\mathbf{n}}$.

Let $\mathcal{L}=\left\{\ell: \mathbf{R}^{n} \rightarrow \mathbf{R}^{n}, \ell\right.$ is linear, $\left.\ell \neq 0\right\}$. Then $\mathcal{L}$ can be identified with $\mathbf{R}^{n}-\{0\}$. We have:

Lemma 2. For almost all elements $\ell$ of $\mathcal{L},\left.\ell\right|_{V}$ is a Morse function on $V$.

Proof. Let $\eta: V \rightarrow S^{n-1}$ be the Gauss Map. Then from Sard's Theorem we get that the set of critical values of $\eta$ has measure zero in $S^{n-1}$. For $\ell \in \mathcal{L}$, $\left.\ell\right|_{V}$ is not a Morse function on $V$ if and only if $\frac{\nabla \ell}{\|\nabla \ell\|}$ is a critical value of $\eta[4]$.

Definition. For $f\left(x_{1}, \ldots, x_{n}\right)$ a real polynomial of degree $d, d \geq 1$, the bordered Hessian, $B H(f)$, of $f$ is the following $(n+1) \times(n+1)$ real symmetric matrix

$$
B H(f)=\left(\begin{array}{cc}
0 & \nabla f \\
\nabla^{t} f & H(f)
\end{array}\right) \text {, where } H(f) \text { is the Hessian matrix of } f
$$

Let now $a=\left(a_{1}, \ldots, a_{n}\right) \in \mathbf{R}^{n}-\{0\}$, and consider the linear function $\ell(x)=<$ $a, x>$. Let $L=\left.\ell\right|_{V}$, and $p$ a critical point of $L$. We may suppose $f_{n}(p) \neq 0$. Then if $h=\ell-\lambda f$, where $\ell_{i}=\lambda f_{i}, i=1, \ldots, n . p$ is a non-degenerate critical point of $L$ if and only if the following matrix $N$ is non-singular

$$
N=\left(\begin{array}{cc}
0 & \nabla f \\
\nabla^{t} f & H(h)
\end{array}\right)=\left(\begin{array}{cc}
0 & \nabla f \\
\nabla^{t} f & -\lambda H(f)
\end{array}\right)[4]
$$

Since $\nabla \ell=a$, and therefore $\lambda \neq 0$, Lemma 2 implies the following: 
Corollary 2. For $a, \ell, L$ as above, $L$ is a Morse function on $I$ if and only if $\frac{a}{\|a\|}$ does not belong to the image of the set $\Delta=(\operatorname{det} B H(f)=0) \cap V$ under the Gauss map $\eta$.

\section{REFERENCES}

1. GANTMACHER, F. R. The Theory of Matrices, Vols. I, II, Chelsea, 1060.

2. MACLANE, S., BIRKHOFF, G. Algebra, Macmillan, 1970.

3. MILNOR, J. Morse Theory, Annals of Math. Studies, Princeton University Press, \# 51.

4. SAKKALIS, T. "An Algorithmic Application of Morse Theory to Real Algebraic Geometry," Ph.D. Dissertation, University of Rochester, $19 S 6$. 


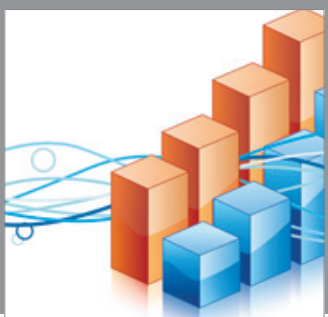

Advances in

Operations Research

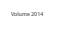

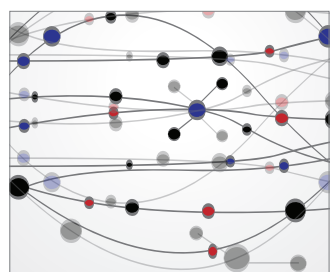

\section{The Scientific} World Journal
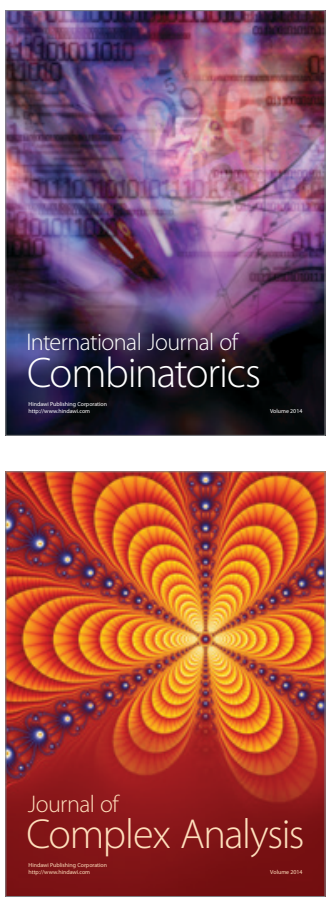

International Journal of

Mathematics and

Mathematical

Sciences
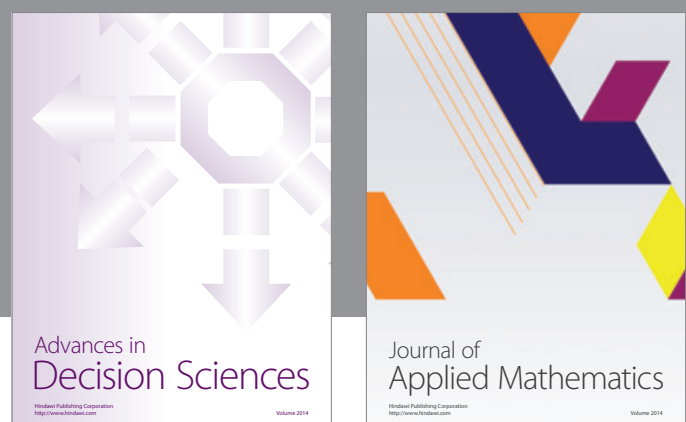

Journal of

Applied Mathematics
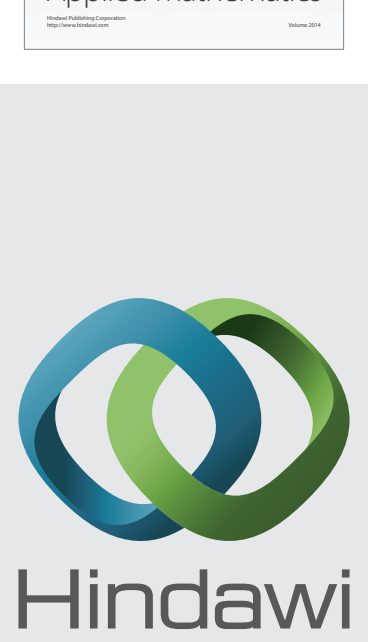

Submit your manuscripts at http://www.hindawi.com
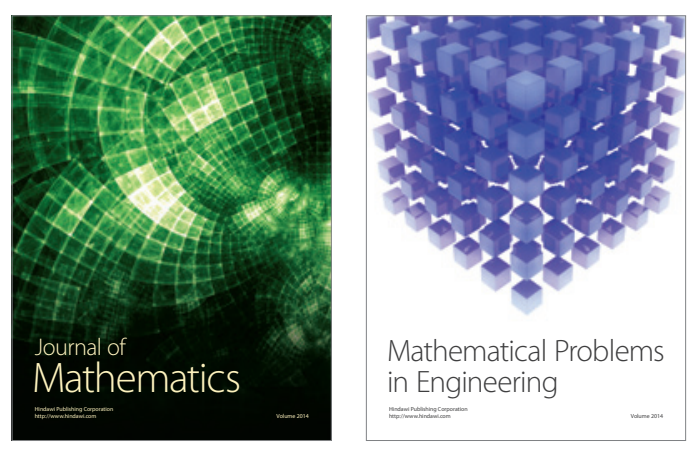

Mathematical Problems in Engineering
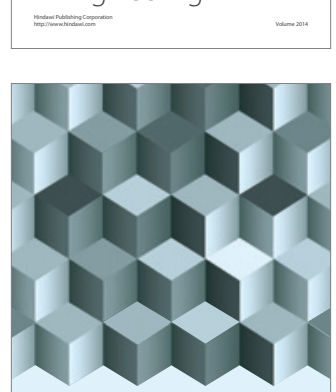

Journal of

Function Spaces
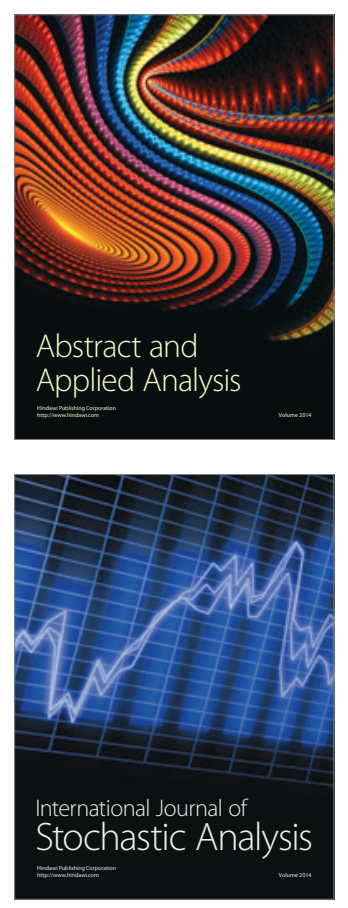

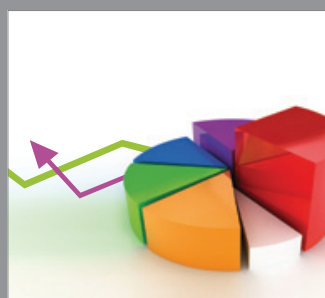

ournal of

Probability and Statistics

Promensencen
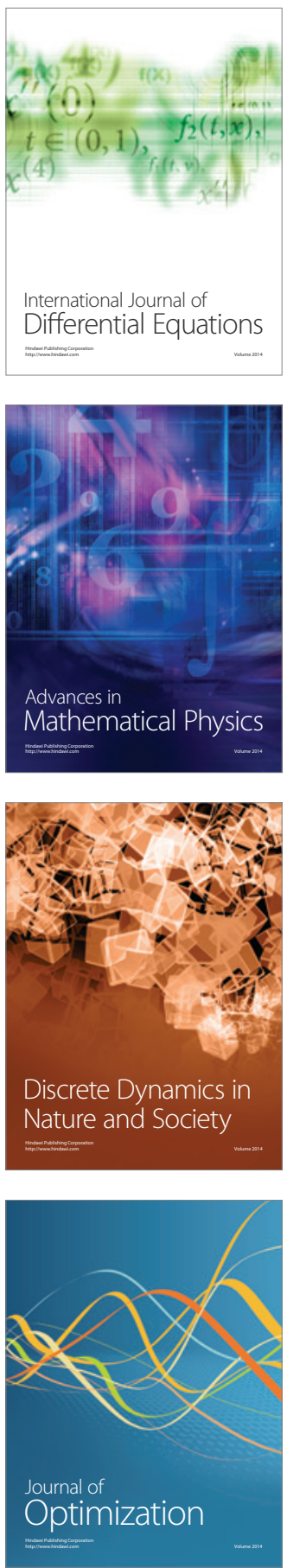